\title{
Influence of Thioctic Acid on the Hyperinsulinemia and Ovarian Volume in Female Patients with Polycystic Ovary Syndrome
}

\author{
L. A. Ivanova \\ Endocrinology Department, Kuban State Medical University, Krasnodar, Russia \\ Email: endocrinkgmu@mail.Ru
}

Received 16 February 2015; accepted 3 April 2015; published 9 April 2015

Copyright (C) 2015 by author and Scientific Research Publishing Inc.

This work is licensed under the Creative Commons Attribution International License (CC BY).

http://creativecommons.org/licenses/by/4.0/

(c) (7)

Open Access

\begin{abstract}
45 female patients with polycystic ovary syndrome took thioctic acid (Thioctacid-HR), $600 \mathrm{mg}(\mathrm{n}=$ $25)$ or high protein diet $(n=20)$. Fast insulin and glucose stimulus insulin were investigated before and after 3 months taken treatment. The use of thioctic acid, $600 \mathrm{mg}$ is a new effective pathogenetics therapy of polycystic ovary syndrome on influence of hyperinsulinemia, HOMA-IR index and ovary volume in female patients with polycystic ovary syndrome.
\end{abstract}

\section{Keywords}

\section{Thioctic Acid, Hyperinsulinemia, Polycystic Ovary Syndrome}

\section{Introduction}

Many researchers believe that hyperinsulinemia is the main cause of the development of polycystic ovary syndrome (PCOS). The group of insulin sensitizers (biguanides, glitazones) has now been successfully used for treatment of hyperinsulinemia in patients with PCOS, contributing to the normalization of the menstrual cycle and fertility in more than half of the patients. Thioctic acid (Thioctacid-HR) can reduce hyperinsulinemia in men and women with obesity, leading to body weight decrease, insulin resistance, improvement of lipid and carbohydrate metabolism.

The objective is to evaluate the effect of alpha-lipoic acid (Thioctacid-HR) on hyperinsulinemia, insulin resistance, menstrual function, ovarian volume in patients with PCOS. 


\section{Material and Methods}

The study included 25 women with PCOS aged 18 to 35 years (average age $25.4 \pm 2.5$ years) who formed the experimental group. Diagnosis of PCOS was conducted according to the recommendations proposed in 2003 by Joint Congress of the European Society of Human Reproduction and the American Society for Reproductive Medicine. After the initial survey patients of the experimental group received thioctic acid (Thioctacid-HR) 1 tablet 30 minutes before breakfast for 3 months and assigned to high-protein diet combined with total exception of digestible carbohydrates in pure form. The control group consisted of 20 women with PCOS (average age $23.7 \pm 2.2$ years, average body weight $29.4 \pm 0.2 \mathrm{~kg} / \mathrm{m}^{2}$ ), who kept to a triple high-protein diet and total exception of digestible carbohydrates in pure form. The groups were matched by age, the incidence of pre-obese and obesity, carbohydrate and lipid metabolism disorder.

The exclusion criteria were pregnancy, taking oral contraceptives within 3 months before the study, severe somatic diseases.

The study protocol included: evaluation of basal rectal temperature for 3 menstrual cycles, at baseline and after 3 months; Measurement of body weight, body mass index (BMI), and determination of blood hormone levels by radioimmunoassay method (on $3^{\text {rd }}-5^{\text {th }}$ and $21^{\text {st }}-24^{\text {th }}$ days of the menstrual cycle) of follicle stimulating hormone (FSH), luteinizing hormone (LH), total testosterone (T), estradiol (E2), dehydroepiandrosterone sulfate (DHEA-S), prolactin (PRL), total cortisol, thyroid stimulating hormone (TSH).

Initially 17-oxyprogesterone (17-OHP) was investigated and if the values exceeded laboratory norm, the ACTH stimulation test (with Synacthen depot, $1 \mathrm{mg}$ ) was performed. In identifying the increase of 17-OHP in three times or more, genetic search of 21-hydroxylase deficiency was conducted. Initially and after 3 months of study transvaginal ultrasonography of the pelvic organs on a 3 - 5 days of ovarian-menstrual cycle was conducted.

Hyperinsulinemia was detected on the basis of an oral glucose tolerance test with $75 \mathrm{~g}$ of pure glucose (OGTT). The presence of insulin resistance (IR) was judged by the index HOMA (fasting glucose (mmol/l) $\times$ fasting IRI (mU/L)/22.5). Impaired glucose tolerance (IGT) and impaired fasting glucose (IFG) was diagnosed according to WHO recommendations, 2001.

Clinico hormonal characteristics of patients are shown in the Table 1.

Table 1. Initial characteristics of women with PCOS $(n=45)$.

\begin{tabular}{ccc}
\hline Index & Experimental group $(\mathrm{M} \pm \mathrm{m})$ & Control group $(\mathrm{M} \pm \mathrm{m})$ \\
\hline n (number of patients) & 25 & 20 \\
Age (years) & $23.9 \pm 1.9$ & $25.4 \pm 2.2$ \\
BMI (kg/m²) & $30.7 \pm 0.6$ & $30.7 \pm 0.6$ \\
Fasting plasma glucose (mmol/l) & $4.8 \pm 0.09$ & $147.3 \pm 7.2$ \\
Basal insulin (pmol/l) & $137.3 \pm 5.6$ & $579.2 \pm 8.7$ \\
Stimulated insulin (pmol/l) & $591.4 \pm 9.1$ & $5.9 \pm 1.0$ \\
HOMA-IR & $5.7 \pm 1.0$ & $0.97 \pm 0.07$ \\
Testosterone (ng/ml) (n = 8) & $0.89 \pm 0.05$ & $216 \pm 23.4$ \\
Estradiol (pmol/l) & $239 \pm 25.9$ & $4.86 \pm 0.08$ \\
Progesterone (nmol/) & $4.06 \pm 0.08$ & $2.60 \pm 0.16$ \\
LH/FSH & $2.50 \pm 0.13$ & $2.13 \pm 0.11$ \\
17-OHP (ng/ml) & $2.87 \pm 0.15$ & $11.1 \pm 1.6$ \\
Average ovarial volume, ml & $12.4 \pm 1.9$ & $20(100 \%)$ \\
Hyperinsulinemia, n (\%) & $25(100 \%)$ & $7(33 \%)$ \\
BMI $>$ 25 kg/m ${ }^{2}, \mathrm{n}(\%)$ & $8(33 \%)$ & $5(25 \%)$ \\
Androgenic dermopathy, n (\%) & $7(28 \%)$ & $6(30 \%)$ \\
Hyperlipidemia, n (\%) & $6(24 \%)$ & $2(10 \%)$ \\
Acanthosis nigricans, n (\%) & $4(16 \%)$ & $1(5 \%)$ \\
IGT, n (\%) & $3(12 \%)$ & $2(10 \%)$ \\
IFG, n (\%) & $2(8 \%)$ & - \\
\hline
\end{tabular}

Note: Here and below: BMI_body mass index, HOMA—IR-insulin resistance index, LH_luteinizing hormone, FSH—follicle stimulating hormone, IGT-impaired glucose tolerance, IFG-impaired fasting glycemia. 
Statistical data processing was performed by Statistica for Windows version 6.0. The reliable values were $\mathrm{p}<$ 0.05 .

\section{Results of the Study}

The majority of patients $(n=45)$ included in the study were characterized by menstrual irregularities, which in 20 patients (44.5\%) were manifested with oligomenorrhea or opsomenorrhea, in 9 (20\%)—amenorrhea, 14 patients (32\%) — had anovulatory cycles. In all studied women basal hyperinsulinemia $(n=21)$, post-prandial hyperinsulinemia $(n=12)$ or their combination $(n=12)$ occured. Four patients were confirmed to be heterozygous carriers of 21-hydroxylase gene mutation.

As a result of treatment with thioctic acid (Thioctacid-HR) $600 \mathrm{mg} /$ day for 3 months there was a significant decrease in average basal insulin $104.7 \mathrm{pmol} / \mathrm{L}(67 \%, \mathrm{p}=0.023)$ and a decrease in average postprandial insulin $499.6 \mathrm{pmol} / \mathrm{L}(54.4 \%, \mathrm{p}=0.015)$. In the control group, the levels of the average values, in both basal and postprandial insulin were practically unchanged $(\mathrm{p}=0.761)$.

Taking of thioctic acid (Thioctacid-HR) at a dose of $600 \mathrm{mg}$ per day resulted in a significant decrease in the average index of insulin resistance after 3 months of treatment $(p=0.038)$ compared with the original data. Among patients, treated with high-protein diet only, the average index of insulin resistance remained practically unchanged $(p=0.83)$ (Figure 1).

Thioctic acid (Thioctacid-HR) treatment at a dose of $600 \mathrm{mg} /$ day improved menstrual function and favorably affected the average ovarian volume after 3 months of treatment. Among patients, treated with Thioctacid-HR in $20(80 \%)$ the duration and frequency of ovarian-menstrual cycle was normalized. Every third patient with amenorrhea were induced menstruation, two patients had dominant follicle. Normalization of the average ovarian volume after 3 months of treatment occurred in 16 (64\%) patients of the thioctic acid group (Figure 2).

In the control group, similar positive results after 3 months of treatment were absent.

All three women - heterozygous carriers of 21-hydroxylase gene mutation-had the same positive effects of influence of thioctic acid on hyperinsulinemia, ovarial volume, HOMA-IR, restoration of ovulatory cycles like patients in the main group.

\section{Discussion}

The results of our study showed that taking of thioctic acid (Thioctacid-HR) at a dose of $600 \mathrm{mg}$ per day resulted in a significant decrease in basal and/or glucose-stimulated hyperinsulinemia, as well as a decrease in insulin resistance index in women with PCOS and heterozygous carriers of 21-hydroxylase gene mutation compared with the high-protein diet. Thioctacid-HR at a dose of $600 \mathrm{mg}$ /day significantly reduces the average volume of the ovaries and has a positive effect on menstrual function in women with PCOS and heterozygous carriers of 21-hydroxylase gene mutation after 3 months of treatment (Figure 3).

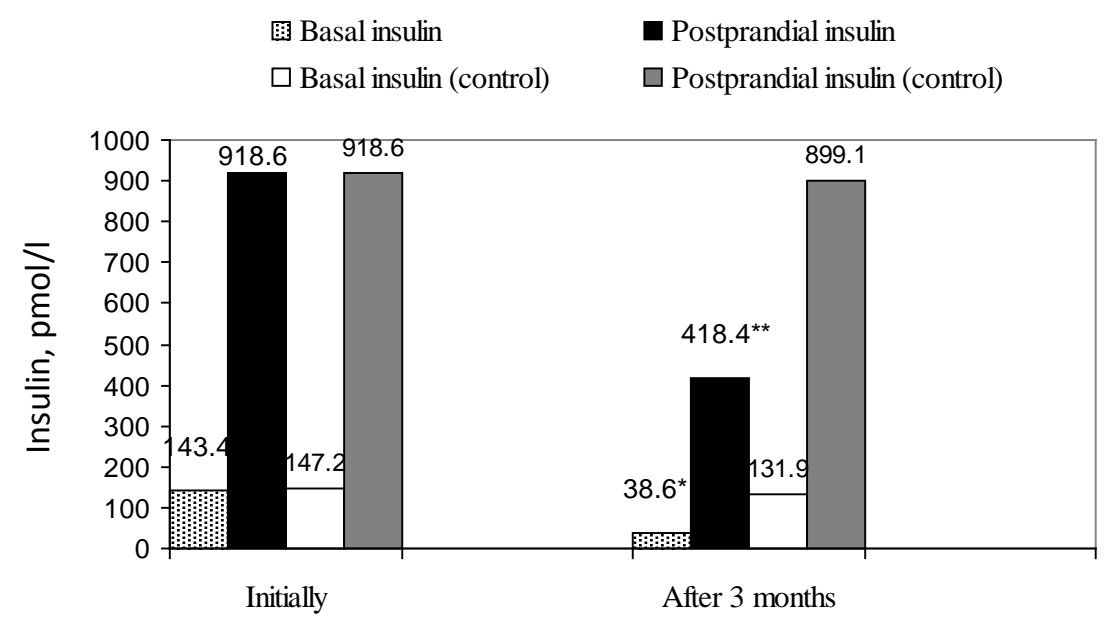

Figure 1. Influence of thioctic acid $600 \mathrm{mg} /$ day on insulin level $(\mathrm{n}=25){ }^{*} \mathrm{p}=0.015 \mathrm{ac}-$ curacy in comparison with the original data; ${ }^{* *} \mathrm{p}=0.023$ accuracy in comparison with the original data. 
- Thioctic acid $(\mathrm{n}=25)$ 橉 Control $(\mathrm{n}=20)$

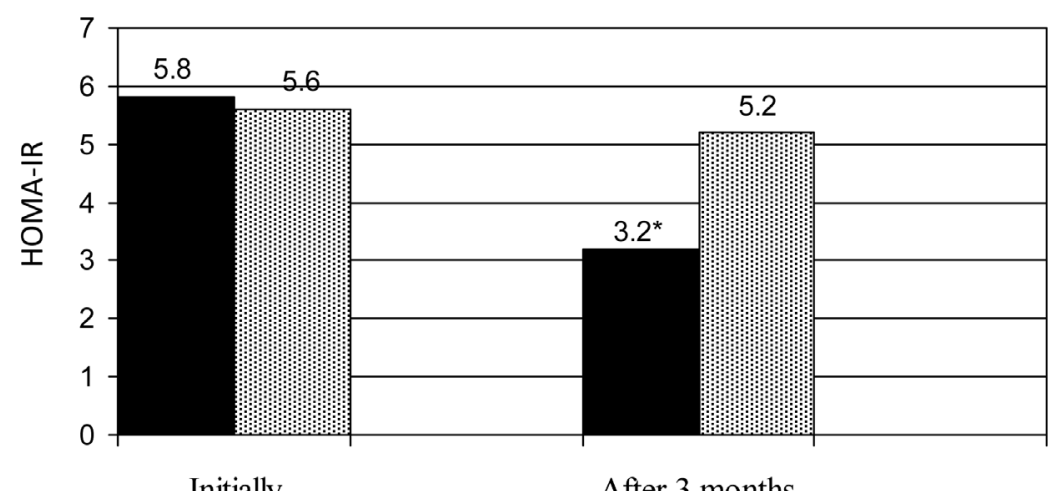

Figure 2. Influence of thioctic acid $600 \mathrm{mg} /$ day on insulin resistance index (HOMA-IR). ${ }^{*} \mathrm{p}=0.018$ accuracy in comparison with the original data.

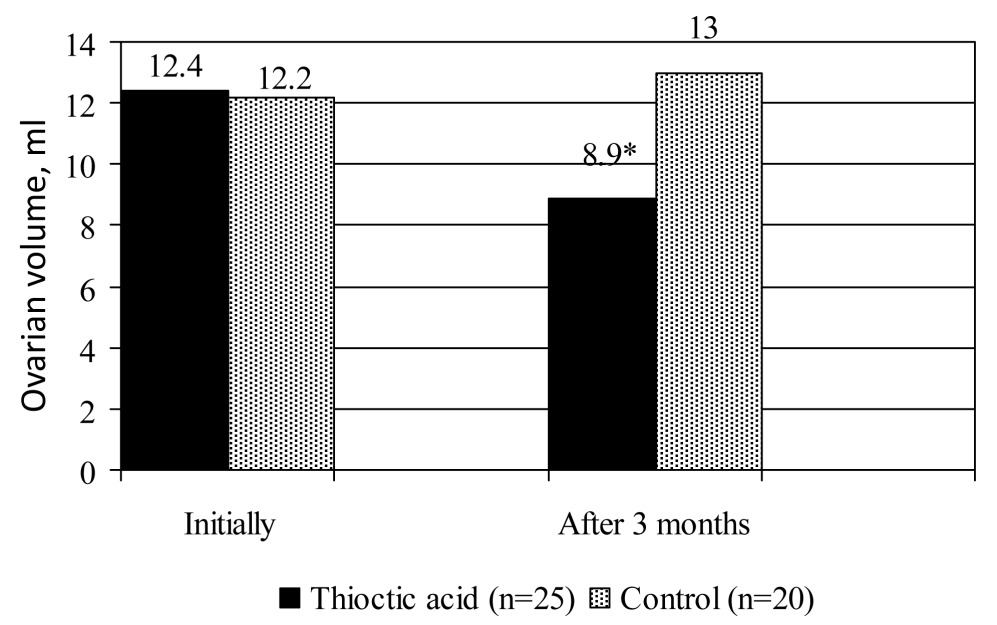

Figure 3. Influence of thioctic acid $600 \mathrm{mg}$ /day on average ovarian volume. ${ }^{*} \mathrm{p}<0.005$ accuracy in comparison with the original data.

Our findings are comparable to the effects of glitazones biguanide described in the literature [1]-[6], which are limited in patients with liver disease [3]. Thioctic acid, on the other hand, is long known as an effective hepatoprotector [2], so it may be the medicine of choice in patients with polycystic ovary syndrome and hyperinsulinemia in combination with liver disease (hepatitis, cirrhosis, decompensated steatosis).

\section{References}

[1] Ivanova, L.A. (2004) Comparison of Treatment of Adiposity by Different Methods. International Journal of Obesity and Related Metabolic Discorders: 13th European Congress on Obesity, 28, 132.

[2] Holte, J. (1998) Polycystic Ovary Syndrome and Insulin Resistance: Thrifty Genes Struggling with Overfeeding and Sedentary Life Style. Journal of Endocrinological Investigation, 21, 589-601.

[3] Ivanova, L.A. (2007) Thioctic Acid and Hormonal Replacement Treatment in Men and Women with 2 Type Diabetes and Obesity. Journal of Clinical Lipidology, 1, 499.

[4] Ivanova, L.A. (2011) Influence of Thioctic Acid on Metabolic Syndrome. 4th International Congress on Prediabetes and the Metabolic Syndrome, Madrid, 6-9 April 2011, 92.

[5] Ivanova, L.A. (2009) Influence of Thioctic Acid on the Metabolic Parameters during Long Treatment of Diabetic Peripheral Neuropathy in Type 2 Diabetics. ISDN/Neurodiab Conference, Canada, 20-21.

[6] Palomba S. et al. (2008) Role of Metformin in Patients with Polycystic Ovary Syndrome: The State of the Art. Minerva Ginecologica, 60, 77-82. 